\title{
GridBox pilot project
}

\section{A platform for monitoring and active control of distribution grids}

\author{
Alain Brenzikofer ${ }^{1}$ - Florian Müller ${ }^{1}$ - Alexandros Ketsetzis ${ }^{2}$ - Florian Kienzle $^{2}$. \\ Marco Mangani $^{2}$ - Marc Eisenreich ${ }^{3}$ - Yamshid Farhat ${ }^{3}$ - Rainer Bacher ${ }^{4}$
}

Published online: 19 July 2016

(C) The Author(s) 2016. This article is published with open access at Springerlink.com

\begin{abstract}
GridBox is an open platform for monitoring and active control of distribution grids. It is based on an innovative concept that comprehensively addresses the challenges DSOs will be exposed to in the context of increasing amount of decentralized and often fluctuating generation as well as the electrification of the heat and transportation sector. In this paper, we outline the principles of the GridBox concept, we describe its key elements in terms of hardware and software and we specify functionalities and applications. The practical implementation of the concept is illustrated by presenting an overview of the results from field tests in two different regions in Switzerland one in an urban grid area in the city of Zurich and one in a rural grid area in the canton of Bern. Results from the evaluation campaigns for state estimation and optimization algorithms are presented.
\end{abstract}

Keywords Distribution grid · State estimation .

Optimal power flow $\cdot$ Topology estimation

\section{Motivation}

With the increasing amount of decentralized and often fluctuating generation and the electrification of the heat and

The GridBox pilot project was co-funded by the Swiss Federal Office of Energy.

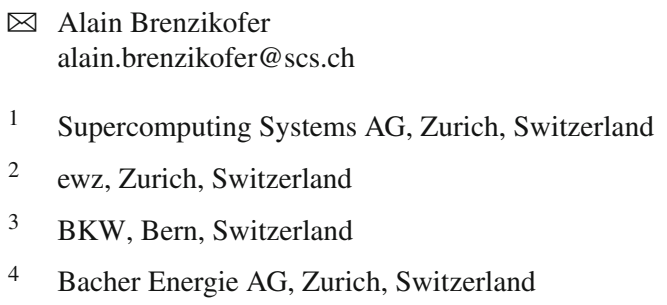

transportation sector, the planning and operation of distribution grids has to be adapted. DSOs will have to balance their investment in the new network infrastructure and in smart technology for active network management to ensure a stable operation of the grid in a cost-efficient way. In addition, the regulatory requirements for distribution system operators necessitate more and more detailed documentation on reliability of supply and power quality. Therefore, DSOs will need better information and control of the state of their distribution grids especially on the medium- and low-voltage level. On these two voltage levels, the usage of monitoring and control devices is currently very limited. As a consequence, the observability and controllability of the distribution grids on these voltage levels is typically low. This represents a big challenge for DSOs as most of the new generation capacities such as photovoltaic (PV) or wind power plants are connected to these voltage levels.

Several studies show the potential impact of distributed generation (DG) capacities on the needs for distribution grid reinforcements and extensions [1,2]. These studies come to the conclusion that the costs of DG grid integration can be significantly reduced by adopting innovative grid components like regulated distribution transformers and line voltage regulators or active/reactive power controlling devices as well as information and communication technologies. It is exactly these issues which the GridBox concept addresses.

\section{The GridBox concept}

The GridBox concept represents an open platform which provides solutions to the challenges mentioned above. At the core of the GridBox concept is a highly distributed network of real-time measurement devices synchronized by GPS. All devices within this network measure voltage and 
current synchrophasors with a high accuracy in real time and communicate the measurement data to hierarchically layered masters. Thus, in contrast to purely decentralized control approaches, the GridBox concept allows for an optimal balance between local control functions and centralized functions being activated by the GridBox master. The centralized monitoring and control is enabled by a continuous grid state estimation carried out by the GridBox master. The state estimation uses the real-time phasor measurements communicated by all GridBox devices in the network. In this way, appropriate control signals can be sent to the actuators of components that are controllable by the GridBox devices in the case of a critical grid state. Examples of grid components that can be controlled in such a way are low voltage regulation systems for controlling the voltage in an individual distribution feeder or controllable local grid transformers with on-load tap-changers. Distributed generation such as PV plants, controllable loads such as water boilers or storage devices such as battery energy storage systems (BESS) can also be controlled by the GridBox devices. Thus, the GridBox platform aims at integrating any type of controllable power generation, load, storage and intelligent building technology and offers a flexible framework for grid monitoring and optimizing algorithms. In this way, the GridBox concept enables an active network management by DSOs. This endows DSOs with the opportunity to optimally balance the use of Smart Grid technologies with conventional grid development measures in the short-, medium- and longterm. Furthermore, the open platform principle shall guarantee interoperability and the seamless integration of third party applications.

\section{Key elements of the Gridbox concept}

The GridBox devices are the basic components of the concept. They measure voltage phasors at the nodes where they are installed as well as current phasors for all lines attached to that node. The form of the GridBox is chosen to fit distribution cabins. Rigid PCB-based as well as flexible Rogowski current sensors have been developed to fit in smaller house connections boxes. The GridBox base module measures voltage on 3 phases and current on 4 phases (R,S,T,PEN). Additional current modules are attached for each line to be measured.

Figure 1 shows the hardware setup for a cable distribution cabinet, to be extended according to the number of feeders present. Apart from measuring voltage and current, the GridBox devices can interface low-voltage relays, DG inverters or storage devices to be able to control load and generation at the grid nodes. Thus, the GridBox devices represent the local physical components of the grid management system.

Another crucial component of the GridBox system is the communication network. GridBox supports several low- as

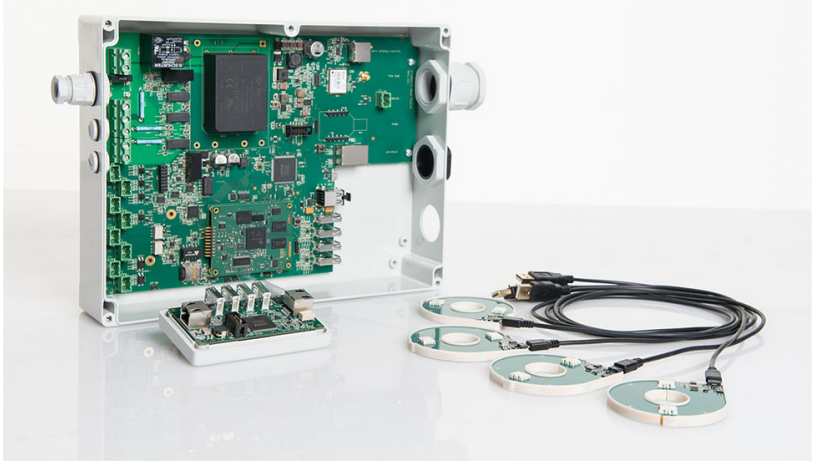

Fig. 1 GridBox base module, current module and Rogowski current sensors

well as high-bandwidth communication channels to minimize operational cost. For the two pilot grids, three communication types are implemented: fibre optics, broadband powerline (BPL) as well as GPRS/UMTS. First experiences have shown that GPRS/UMTS solutions are not suitable for future applications because of their high cost to handle high data volumes. Nowadays, fibre optics are not yet widely available. BPL has a good potential for future application, but is still quite power-hungry and it is restricted by law to grids consisting only of underground cables. Further possibilities are RF transmission in multi-hop mesh networks or narrowband powerline communication (PLC).

Instead of using standardized, generic protocols like IEC 61850, a custom communication protocol based on SCTP needed to be developed to reduce latency, overhead and minimize bandwidth as well as data volume. Moreover, the protocol and data handling needs to be robust against packet loss as GPRS/UMTS as well as BPL are unreliable communication channels. The 'GridNet' protocol is optimized for the transfer of GridBox measurements in intervals of one second and time-limited prioritized retransmission to enforce configurable delay limits and for sending commands to controllable grid components. As an extension, GridBox is designed to allow for protocol tunneling for third party devices. Each grid region is managed by a GridBox master that collects and processes all the data. A real-time state estimation is performed, followed by an optimal power flow algorithm. Those algorithms are described in subsequent sections.

The federalistic approach has several advantages. Data and control is kept as local as possible. This increases robustness and also reduces data security and privacy violation risks. Regions operate semi-independently in a hierarchical way as shown in Fig. 2.

One region only needs to share aggregated grid state indicators with their superordinate region master. So a N7 region represents nothing more than (controllable) prosumers for the N5 region master. This helps reducing data traffic and 


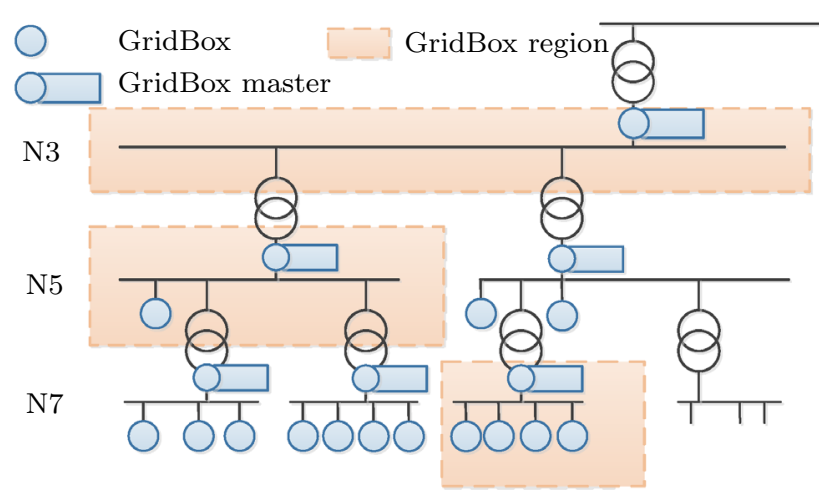

Fig. 2 GridBox system federalistic hierarchy

ensures scalability. However, the two pilot grids only implement one region each with their respective master.

Purely local control of grid-connected devices based on local voltage measurements (as presented in [3]) has the disadvantage that it is not guaranteed to be stable as soon as many such controllers are deployed. When tapchanging transformers or reactive-power-controlled inverters are present, the local voltage level is often an insufficient indicator of the severity of the grid state. A GridBox Master employs regional control based on state estimation of the entire sub-grid to overcome this problem. This concept could eventually be extended to peer-to-peer control without the need for a GridBox master as suggested in [4]. Furthermore, a peer-to-peer alternative of the GridBox project is discussed in [5].

\section{Functionalities and applications}

The core functionality, which has been demonstrated with continuous operation of about 150 devices during 1 year since March 2015, consists of sampling every second the measurement data of each GridBox device and collecting this information in quasi real-time on a GridBox Master. Based on this functionality, two types of applications are possible. On the one hand, real-time applications, so called smart grid applications (SGAs), are executed with the set of information available every second. These include power system state estimation, topology estimation, monitoring and prosumer control. On the other hand, ex post applications that require archived measurement data can be run. The interplay of the SGAs and the archive service within the GridBox system is illustrated in Fig. 3. Here, the measurement accumulator and the governor services provide an interface for the SGAs to receive measurements from and send control signals to the GridBox devices, respectively. Topology estimation of Sect. 4.1 tries to determine the connectivity graph or even the entire model of the corresponding grid. Assuming that a grid model is given, the state estimation of Sect. 4.2 com-

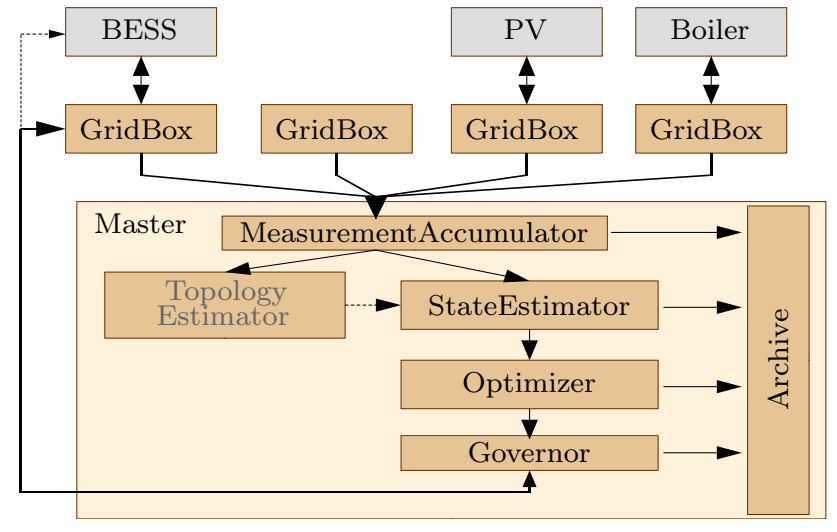

Fig. 3 Smart grid applications (SGA) architecture

pletes and adjusts the GridBox measurements which can be inaccurate or missing altogether. The estimates of the state estimation can then be used to formulate an optimization problem. The solution to this optimization problem determines a set of control signals which, after sending them to the corresponding GridBox devices, improve the overall grid state with respect to a certain objective. This optimal power flow control SGA is discussed in Sect. 4.3. Furthermore, a monitoring SGA and the data archive service are presented in Sects. 4.4 and 4.5, respectively.

\subsection{Topology estimation}

Automated topology identification is a subsequent application which will be tested during the pilot project. The objective is to draw a connectivity graph, i.e. to determine which nodes are connected with each other. Therefore, an algorithm matches node currents on both sides of a line. Especially in a meshed distribution network, the connectivity graph may be subject to frequent changes by switching operations. Even though a distribution system operator normally possesses at least one network diagram for planning and operation purposes, the automated identification can replace manual interventions for the initial input of network data or updates in the case of topology switches. Adding node voltages to the current phasor data used in the topology identification, the electrical parameters of the lines can be obtained so that load flow calculations can be performed. For a discussion of algorithm approaches and results, the reader is referred to $[6,7]$.

\subsection{State estimation}

The elementary real-time application consists of a linear three-phase state estimator based on concepts presented in $[8,9]$ capable of detecting and treating bad data such as measurement deviations and of handling missing data. Missing 


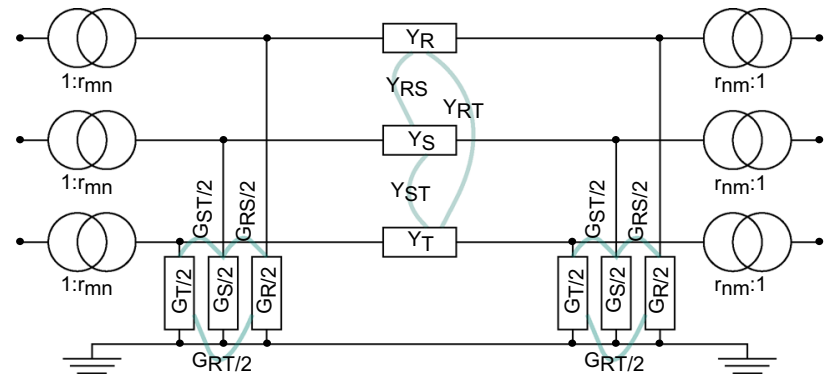

Fig. 4 The line model used for state estimation and optimization. A three-phase pi model with mutual coupling for both series and shunt admittance

data can be of temporary nature in case of communication interruption. However, more relevant are nodes that are not measured at all. The line model used is shown in Fig. 4.

Besides technical reasons such as inaccessible cable junctions in the ground, it is also an economical motivation to deploy only as many GridBoxes as necessary. In both pilot networks, most accessible nodes are equipped with a GridBox device in order to be able to evaluate the confidence metric of the state estimator by deliberately considering only a subset of the available measurement points. Section 6.1 explains the outcomes of this analysis. The state estimation associates to each node estimated values and confidence intervals for current and voltage.

\subsection{Optimal power flow control}

In order to dispatch all controllable prosumers (actors) in an optimal way, an application was developed that solves the three-phase optimal power flow (OPF) problem for the entire grid region with objectives like voltage stability or reducing line or transformer strain at multiple locations in the grid. Further goals could be minimization of grid losses. With that, the DSO will be able to choose among several objective functions.

In order to be reactive enough for voltage stability improvement, the computational load needs to be kept low. This has been achieved by implementing a single-timestep OPF using sequential linear programming (after conducting some preliminary studies based on semidefinite programming, see [10]). Actors with storage capabilities are often controlled using model predictive control (MPC) [11]. As this project focuses on the geographical extent as well as high control reactivity in the order of seconds, such a computationheavy approach has been neglected.

In the pilot grids, the optimizer can control PV plants by curtailing active power or requesting a certain power factor. A BESS located in the ewz pilot grid can be controlled in active as well as reactive power. Water boilers as well as

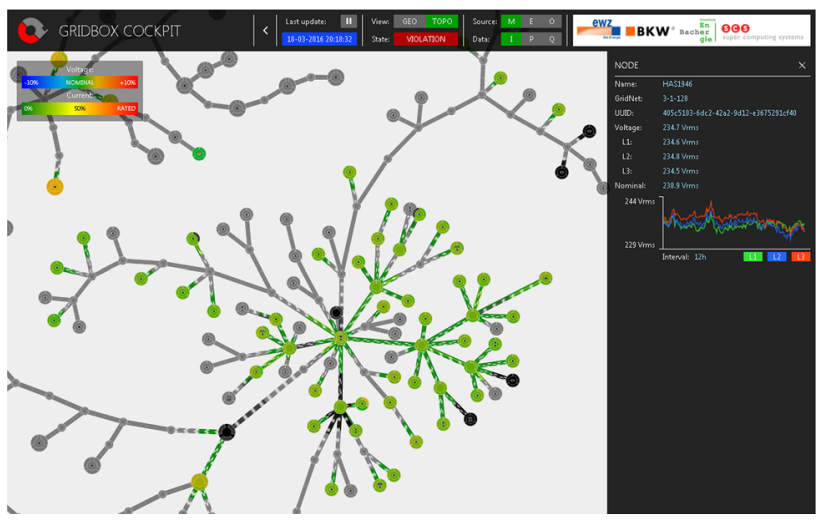

Fig. 5 GridBox Cockpit measurement view showing instantaneous measurement values as well as prosumer states and historical data

heatpumps can be switched on or off in order to shift their load.

\subsection{Monitoring}

The actual state of the distribution network is calculated and evaluated against the permissible operational limits at every instant. To visualize the actual state of the grid as well as constraint violations and historic data, a cockpit has been developed as a web-service as shown in Fig. 5. Three-phase voltages as well as power flows are animated in real time, providing the DSO with a today lacking level of transparency for the lower grid regions.

\subsection{Data archive}

The second major set of possible functionalities can be seen as a by-product of the aforementioned real-time applications. An archive with measured values, like synchrophasors and harmonics of voltages and currents as well as outputs of the real-time algorithms, like estimated values, switching states and optimizer set-point values, can be used to improve existing algorithms and develop new applications with respect to realistic conditions. The synchrophasor measurements could be used to develop sophisticated state estimation algorithms based on machine-learning techniques. As another example, the load and voltage profiles could lead to more efficient network planning. Furthermore, the capability of the GridBox to measure synchronized phasors would allow the implementation of innovative future applications such as fault detection (i.e. locate the exact place where a fault has appeared), transient analysis, detection of unintentional islanding or management and operation of micro-grids (i.e. reconnect a part of the grid without injecting disturbances).

The GridBox platform is designed as open as possible in order to be able to address future questions that are not yet foreseeable today or that require longterm data. 


\section{Development project and field tests}

The GridBox system has been developed within a 3-years research project by the project partners BKW (DSO in the Swiss cantons of Bern and Jura), ewz (DSO of the city of Zurich and part of the Grisons), Supercomputing Systems (development service provider), Bacher Energie (consulting and project support) and co-funded by the Swiss Federal Office of Energy. The GridBox system has been tested in two pilot installations: one in an urban low-voltage grid area in the city of Zurich and one in a rural medium- and low-voltage area in the canton of Bern. In total, roughly 150 GridBox devices have been installed in the beginning of 2015. ewz uses a low voltage grid area in the periphery of Zurich (district Affoltern) for the project. This pilot grid is illustrated in Fig. 6

The chosen area provides opportunities for numerous tests. The goal in the field test is to install GridBox measuring instruments with a complete coverage of all nodes in the network area in order to be able to extensively and reliably validate the concept. As illustrated in Fig. 6, there are several controllable loads and PV plants with controllable inverters in the ewz pilot grid area. GridBox devices can send control signals to the PV inverters so that they provide reactive power for voltage control. Furthermore, tests for active power curtailment of the PV plants are carried out. In addition, there is a battery energy storage system (BESS) installation in the pilot grid area with a power rating of $120 \mathrm{~kW}$ and a nominal capacity of $720 \mathrm{kWh}$.

Most of the GridBox devices at ewz are placed within the distribution grid, either in transformer stations or in distribution cabinets. Some GridBox devices are installed next to house connection boxes. Data security is a crucial issue. The handling of the measured data has to be considered carefully,

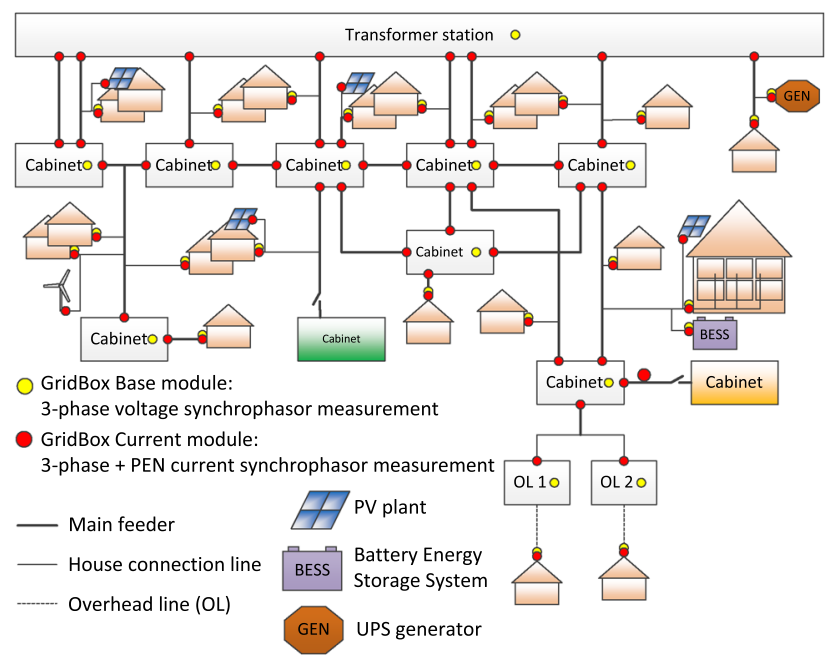

Fig. 6 Urban pilot grid in the city of Zurich (ewz)

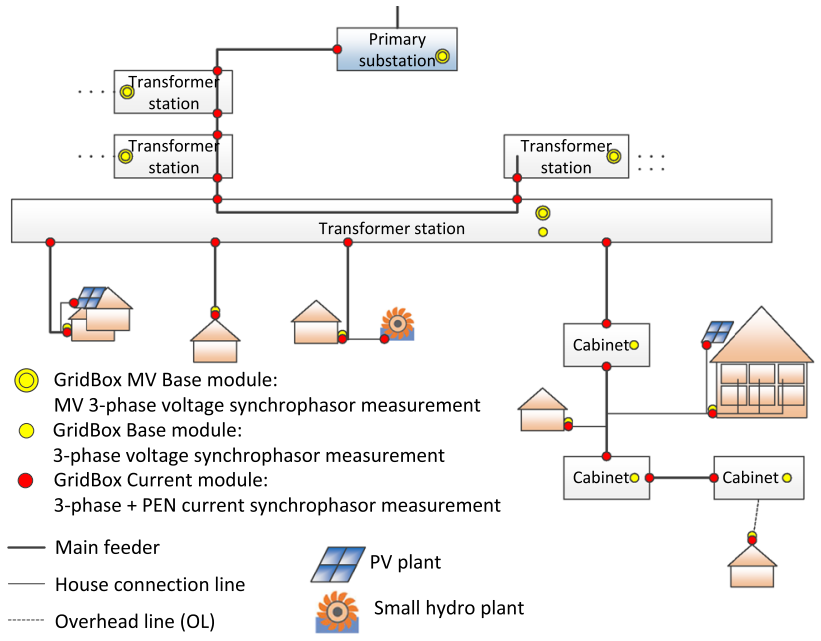

Fig. 7 Rural pilot grid in the canton of Bern (BKW)

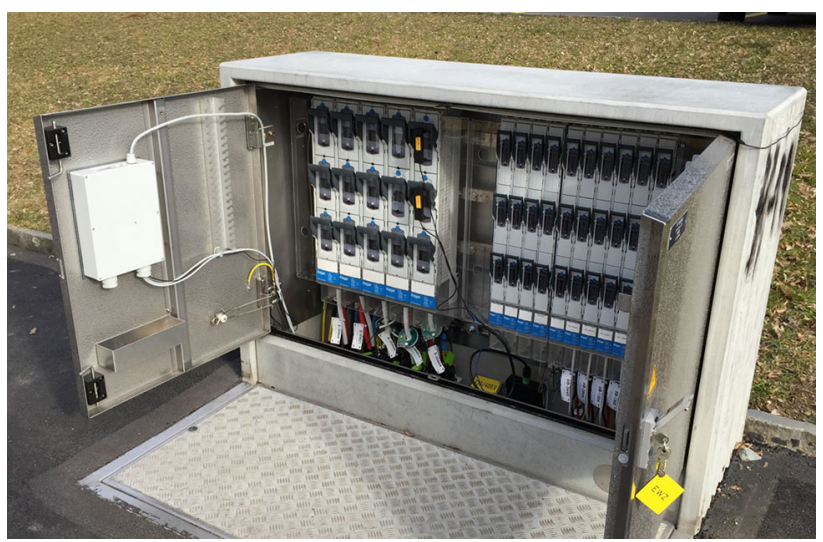

Fig. 8 GridBox installed at a distribution cabin

especially where the GridBoxes are installed at house connection boxes and the measurement data could be attributed to individuals. Therefore, adequate measures for guaranteeing the protection of customers privacy have been adopted.

The BKW pilot network is located in a rural area of the canton of Bern. It comprises a part of the medium voltage network as well as an entire low voltage network (see Fig. 7).

In the low-voltage network, GridBoxes have been installed at almost all accessible points, i.e. on the low-voltage feeders of the transformer stations, the cable distribution cabinets in the streets, and the house connection boxes of the end customers. Figure 8 shows a GridBox installation in a distribution cabinet. In the medium-voltage network, GridBoxes have been installed in selected transformer stations along one substation feeder. While the GridBox base modules can be connected directly to the LV connection points for voltage measurement and power supply, the MV switchgears have been equipped additionally with sensored cable terminations, housing a resistive voltage sensor and a Rogowski-type current sensor. 
At BKW, both voltage levels (MV and LV) are characterized by a radial structure. In accordance with a considerable share of overhead lines and pole-mounted transformers, this network was chosen as it is typical for rural areas. Furthermore several DG units, here photovoltaic systems and one small hydro plant, and controllable loads like water boilers are available. Due to the low customer density in the chosen grid area, the technical options for high volume data communication are very limited. The choice was between the landline DSL and the mobile phone network. In order to be as independent as possible from end customers and because this communication channel is already in use inside the company for remote meter reading, data is sent via the mobile phone network.

Accuracy for all measurement channels was verified by means of a laboratory installation with defined frequency, voltage, current and harmonics content. It can be highlighted that the total vector error for the voltage phasor measurement at nominal frequency and nominal voltage is in the range of $0.04 \%$, dominated by the magnitude error.

\section{Results}

\subsection{State estimator performance}

As the true state of the grid is unknown, there is no way to quantify the accuracy of estimation by itself. The analysis of state estimator performance has therefore been taken out by comparing estimated voltage and current magnitudes to their corresponding measurements. Table 1 shows the accuracy achieved for $95 \%$ of all sensor locations. As shown in [12], model errors are a limiting factor for estimator accuracy, causing a tradeoff between voltage and current accuracy when configuring the state estimator. Further research is needed to develop a model-adaptive state estimator.

The pilot grids have been equipped with a very large number of sensors. For economical reasons it is of interest, how many sensors are needed to achieve a desired accuracy. To answer this question, archived measurement data has been used to re-run different scenarios only using a subset of all available sensor data and comparing the output to the original estimation. Figure 10 shows the degradation of accuracy for different scenarios for the case of the radial BKW low voltage grid shown in Fig. 9. Dropping redundant measure-

Table 1 State estimation accuracy

\begin{tabular}{lll}
\hline & BKW (radial grid) & ewz (meshed grid) \\
\hline Voltage accuracy & $1 \mathrm{Vrms}$ & $2.5 \mathrm{Vrms}$ \\
Current accuracy & $10 \mathrm{Arms}$ & $5 \mathrm{Arms}$ \\
\hline
\end{tabular}

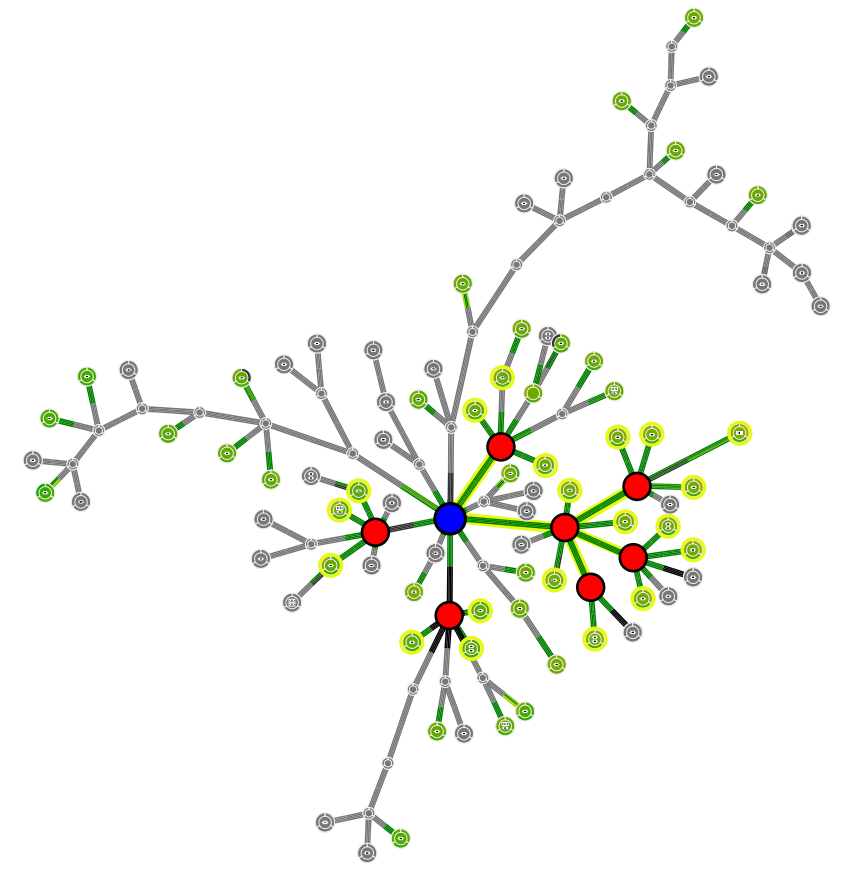

Fig. 9 BKW low voltage grid topology. Household (HAK) measurements are marked in green, distribution cabins (VK) are red the transformer station (TS) is blue. Redundant measurements are highlighted in yellow (color figure online)
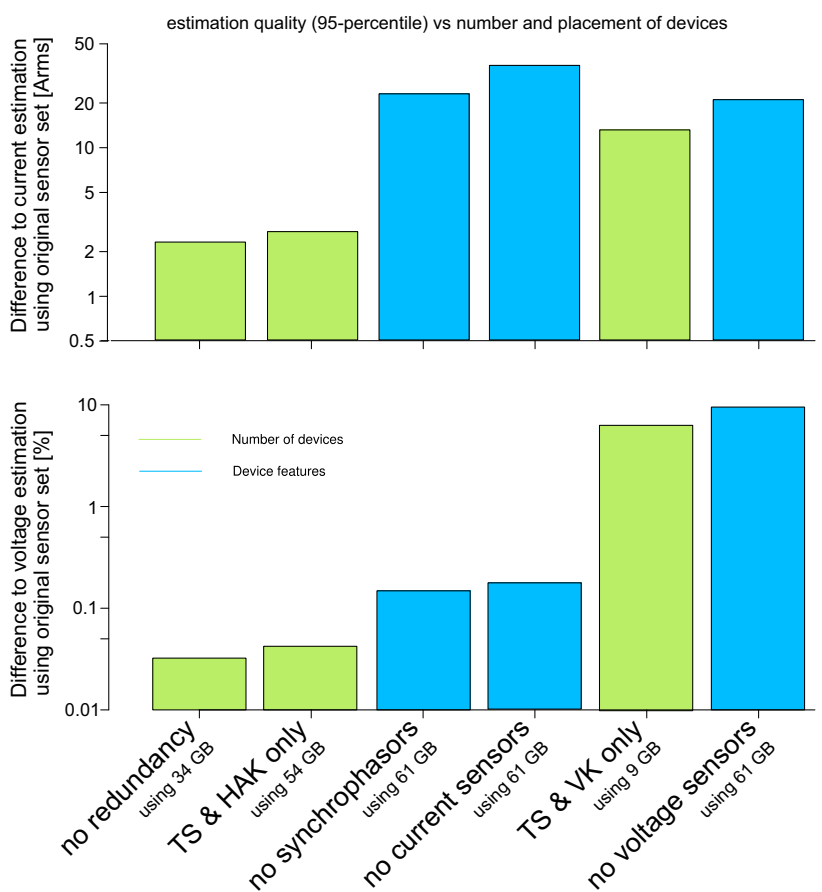

Fig. 10 State estimator accuracy comparison for different sensor placement scenarios. TS, HAK, VK and GB stand for transformation station, Hausanschlusskasten or house connection, Verteilkabine or distribution cabin and GridBoxes, respectively 
ments makes only little difference but allows to use only 34 out of 61 GridBoxes. Omitting measurements at distribution cabins has only little impact (less than $0.5 \%$ ) on voltage accuracy whereas omitting house connection measurements (only using 9 GridBoxes) shows surprisingly good current accuracy (6A) but is weak for voltage accuracy (6\%). Assuming that the measurement devices are not time-synchronized (no-synchrophasors) shows that voltage magnitude estimates depend only little on synchrophasor information, yet the quality of current estimates suffers significantly. Omitting current measurements entirely would be an attractive costsaver on low voltage grids. The effect on voltage accuracy might be acceptable, but if currents are of interest, one should invest on a better voltage measurement accuracy as well as a very accurate grid model. Finally, omitting voltage sensors (except the measurement of the slack voltage) leads to unacceptable voltage accuracy. Further research could apply the same analysis to mid-voltage grids where there is an economical interest to omit voltage sensors in favor of many current sensors. Moreover, additional efforts are needed in order to better understand and explain these results.

\subsection{Voltage stability optimization}

Various campaigns have been taken out to assess the performance of the developed optimizer. The most challenging optimization goal is voltage stability as it requires a very reactive control loop. As shown in [12], the GridBox system
Fig. 11 Undervoltage on one phase during evening times when the optimizer is not running
Fig. 12 Voltages of all three phases during evening time when the optimizer is running
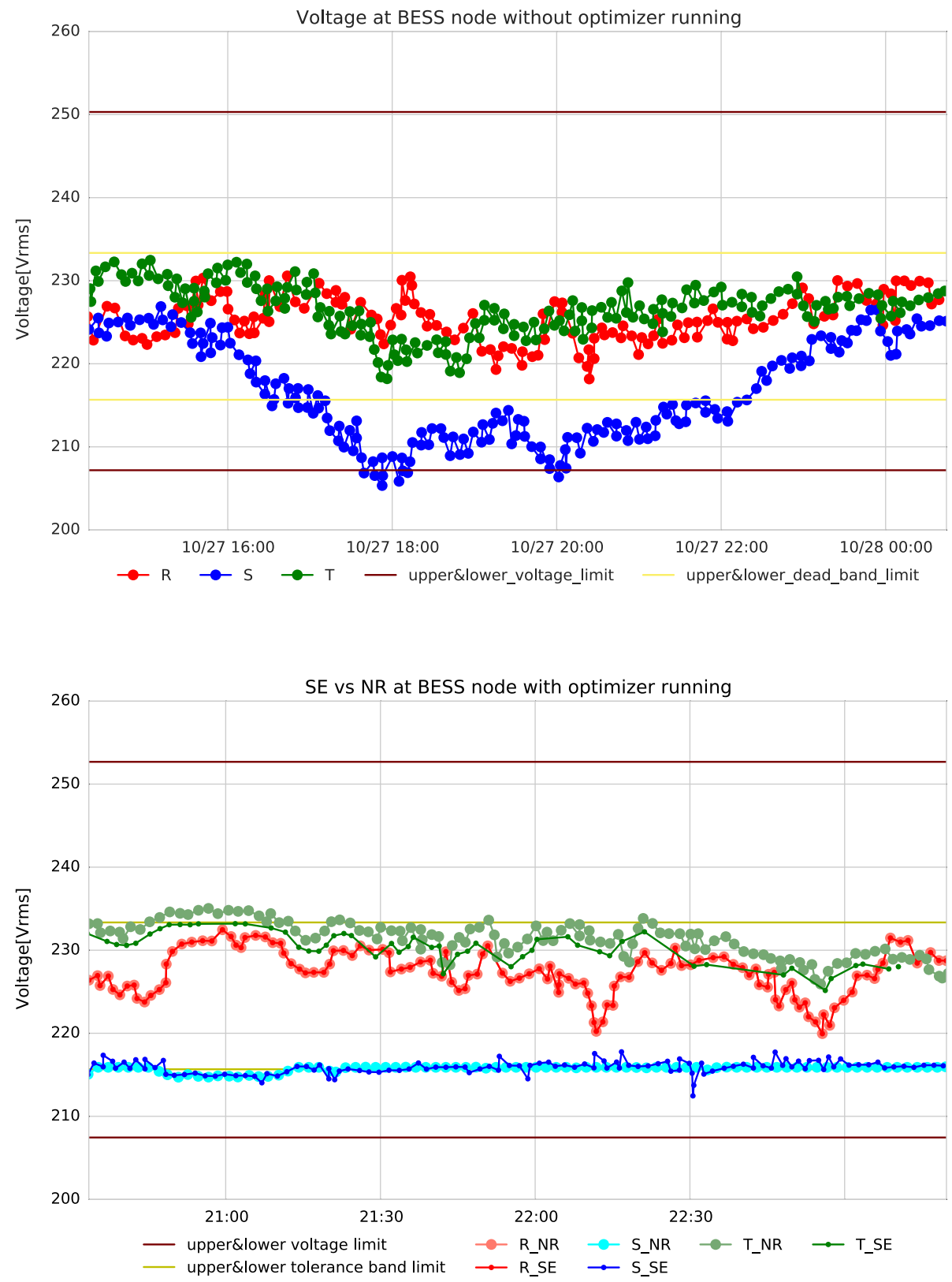
allows to control a prosumer with a lag of 5-12 s, depending on complexity of the objective functions and the size of the grid region.

Figure 11 shows the daily undervoltage events on one of the three phases during evening time at a node in the ewz grid before any optimization takes place. This node hosts a large battery energy storage system (BESS) that can be controlled by the GridBox system. Figure 12 shows that the optimizer is capable of keeping the voltage within a target band. Plotted are voltages reported by the state estimator as well as voltages reported by the optimizer (newton-raphson reconstruction based on optimal prosumer setpoints, to be

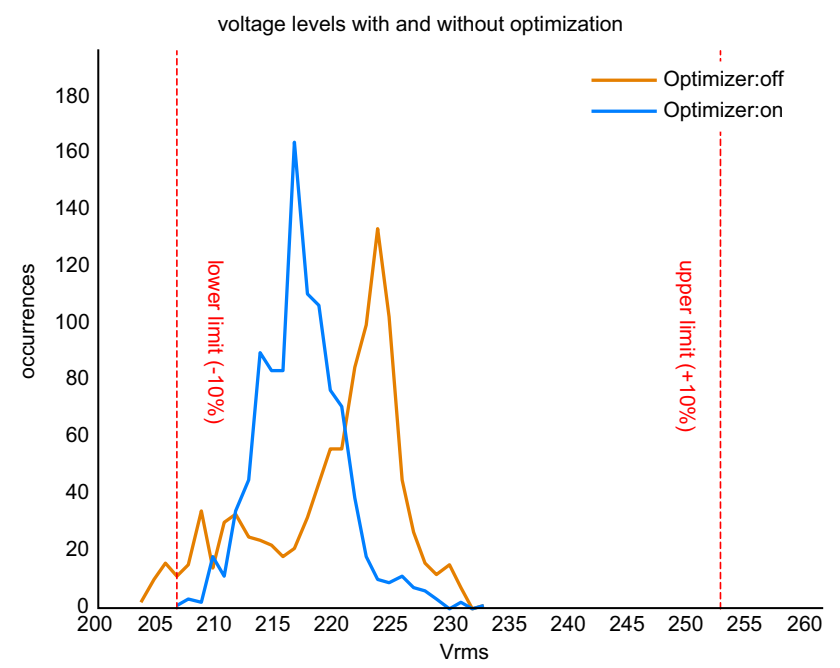

Fig. 13 Histogram of 10-minute-average (according to EN50160) voltage levels with (30.11.2015 until 6.12.2015) and without (30.10.2015 until 5.11.2015) optimization. Undervoltages are successfully mitigated by the optimizer controlling BESS active power understood as a prediction of what will happen as soon as prosumers implement the desired setting). The fact that estimation and prediction stay close together proves that the grid state may be assumed stationary for the duration of our control lag of few seconds. It is worth noting the big voltage difference among the three phases. Unfortunately, the BESS wasn't capable of controlling the phases independently, so mitigating an undervoltage on phase $\mathrm{S}$ led to a slight overvoltage on phase $\mathrm{T}$.

Figure 13 shows the voltage histogram for several days with and without GridBox control. The lower mean value is mainly caused by seasonal changes in electricity demand and only little by battery losses. It can be concluded that GridBox is able to keep the voltages within a desired band by using a BESS.

Controlling active power curtailment and power factor of PV plants has been tested in the BKW grid. Figures 14, 15 and 16 show how the optimizer reacts to overvoltages caused by PV injection. As a first measure, the PV plant is told to 'inject' negative reactive power. Modern PV inverters allow controlling the power factor within the limits of a PF of 0.85 . For the GridBox system it would however be desirable to have the ability to go down to a PF of zero. Active power curtailment is implemented only in the case where the injection of negative reactive power does not mitigate the problem.

Finally, a campaign including all available prosumer types (BESS, PV and boiler) to optimize voltage stability has been carried out. The objective function is a desired voltage band at the node where the BESS and 6 PV plants are installed ( 3 of which are curtailable by GridBox, one is used as an uncontrolled reference plant). Water boilers throughout the grid region are controlled as well. One of the challenges when controlling boilers is the fact that the state-of-charge (SoC) is
Fig. 14 PV campaign: voltage at PV injection node

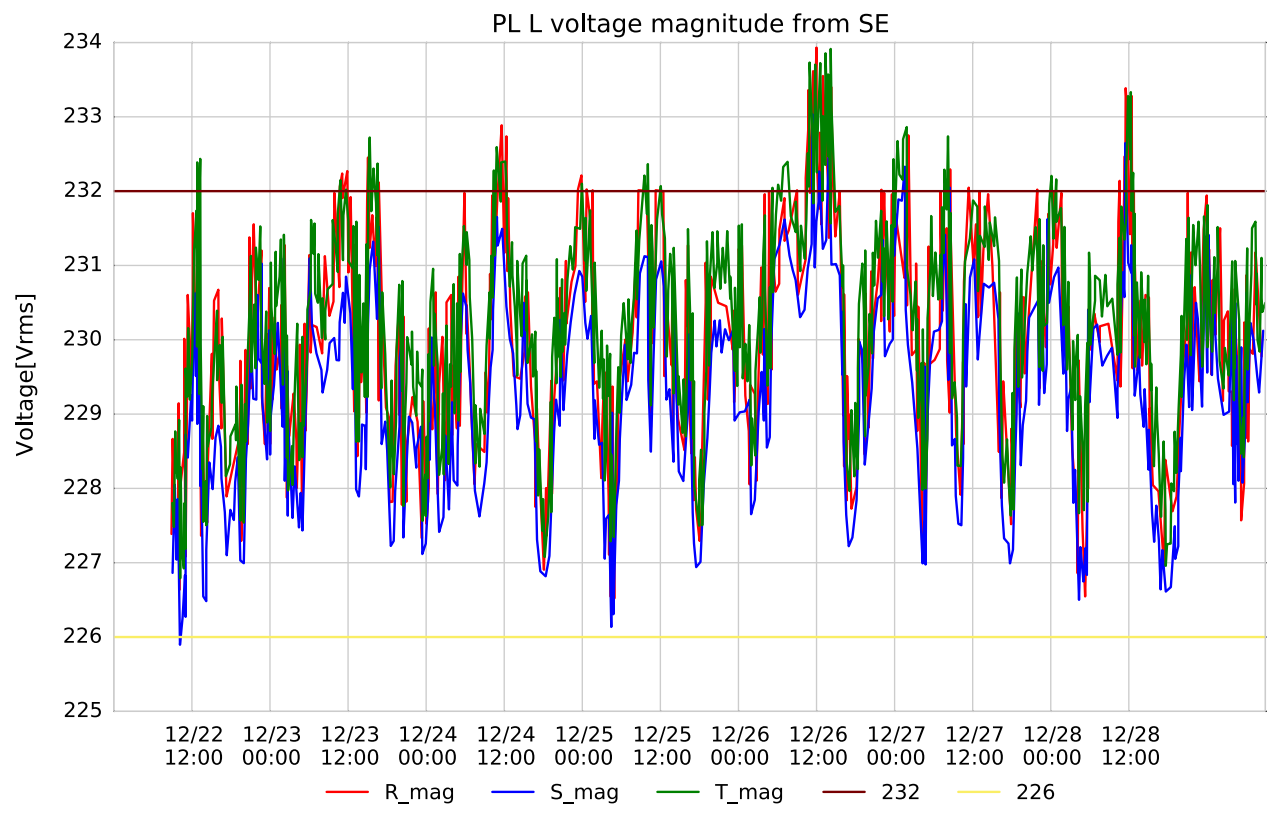


Fig. 15 PV campaign: active and reactive power $\mathrm{P}$ and $\mathrm{Q}$, respectively, at PV injection node

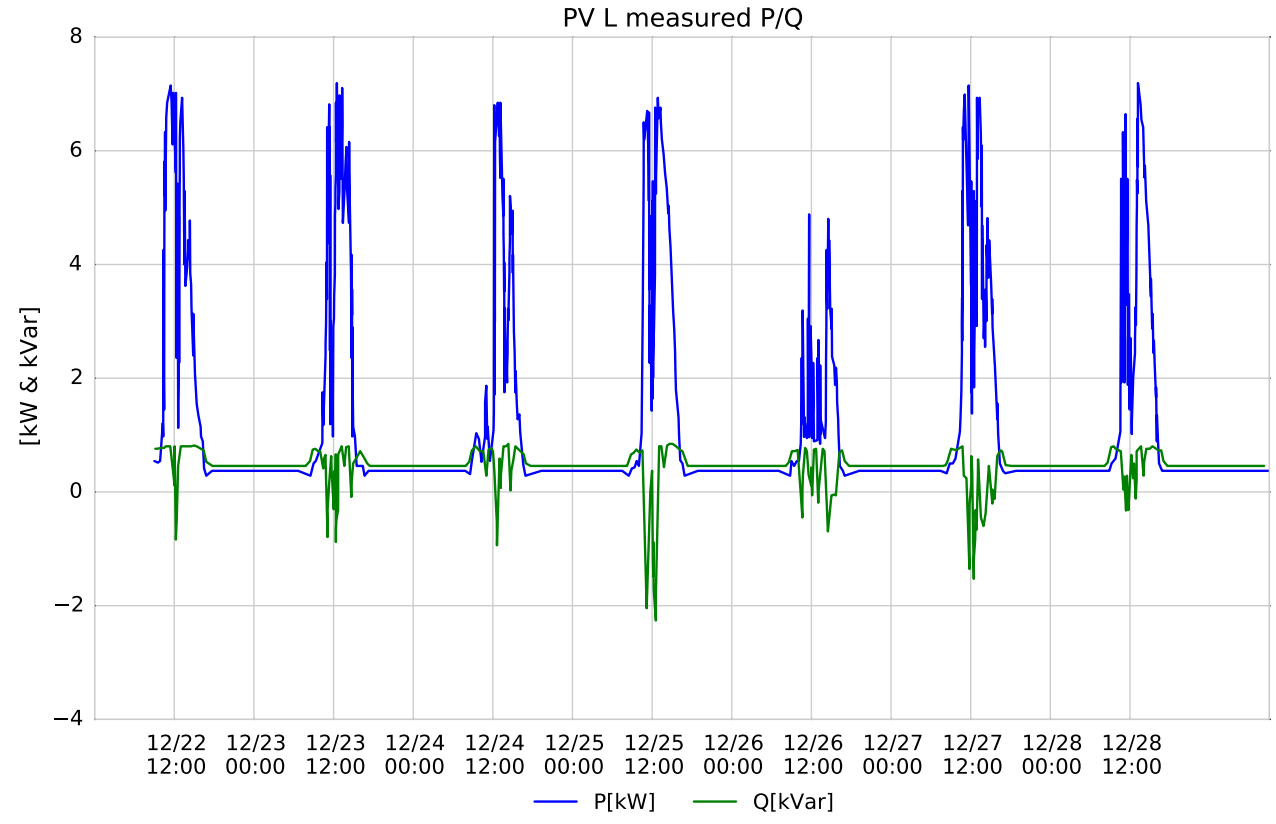

Fig. 16 PV campaign: active control signals in terms of relative active power and phasor angle phi at PV injection node

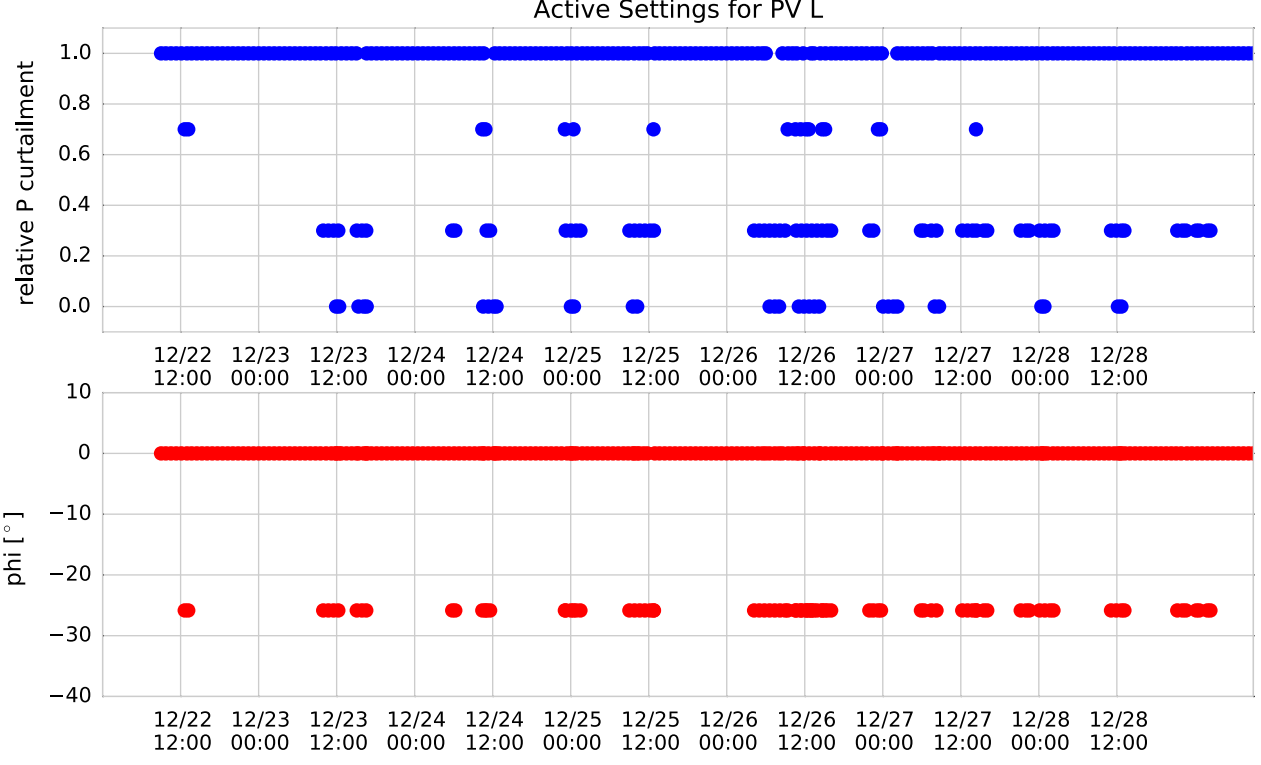

not known. A very simple linear state model has been implemented for that case: the boiler's energy content is charged according to the supplied active power whereas the consumption of hot water is modeled such that in the absence of supply power, a fully charged boiler is depleted within $24 \mathrm{~h}$. Due to limitations of this model and other limitations such as unreliable communications and not taking into account predicted future boiler states, cold showers are prevented by a local security measure implemented on the GridBox devices that can overrule the optimizer to guarantee a minimum on-time for each boiler within $24 \mathrm{~h}$. To compensate for the lack of constraints with respect to predicted future boiler SoCs, a cost term incentivizes boiler SoCs of $50 \%$ at all times with low weight.

Figure 17 shows the voltage at the BESS node during one day of the all-in campaign. The voltage tolerance band for the optimizer is chosen such that both over- and undervoltages occur. As expected with PV plants, there is a rise in voltage around midday. The corresponding PV injections are shown in Fig. 18. The optimizer decided to curtail PV injection to 
Fig. 17 All-in campaign: voltage at the BESS node

Fig. 18 All-in campaign: PV reference irradiation and curtailment
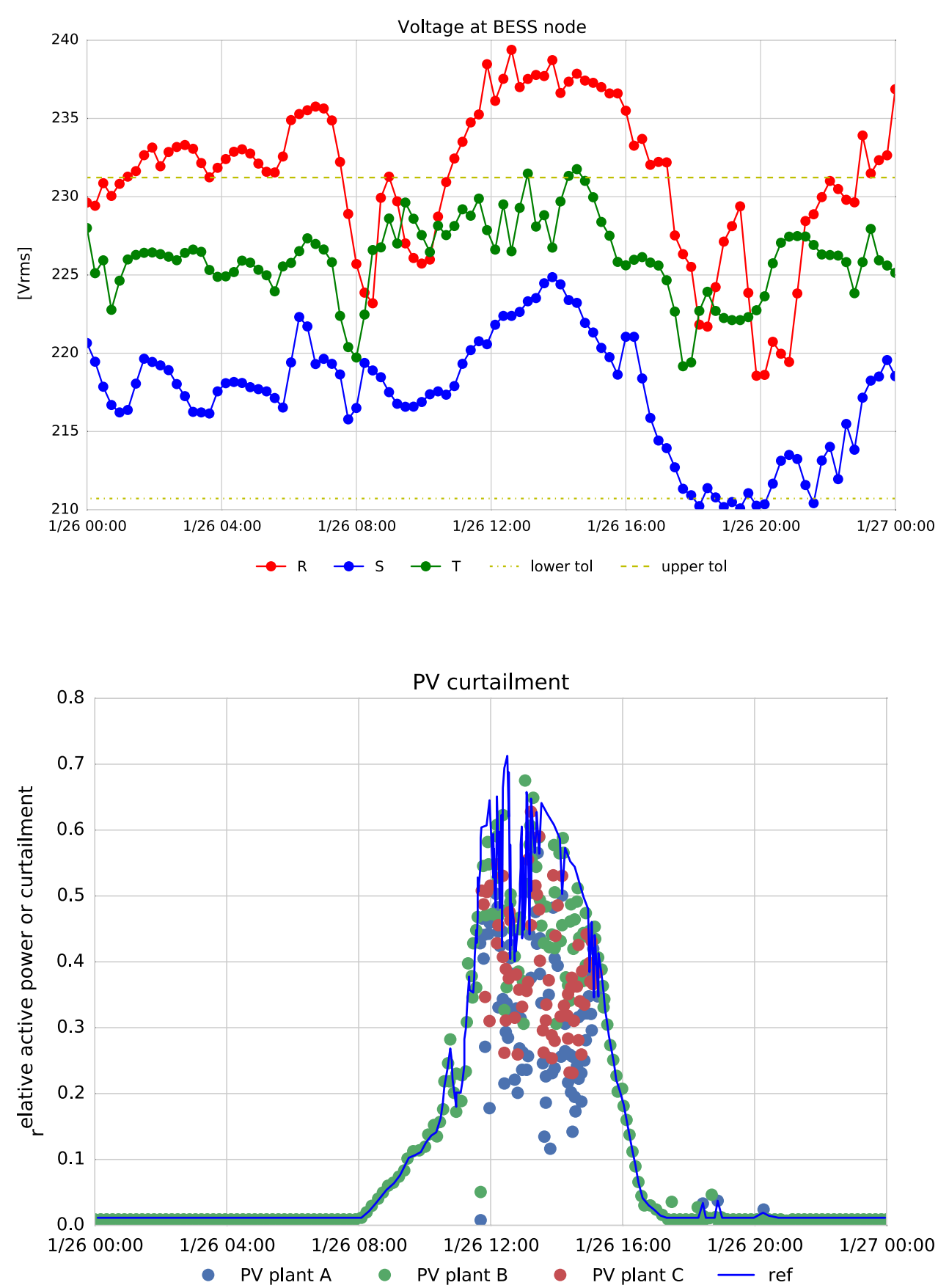

mitigate overvoltages. In order to minimize PV curtailment, the BESS is charging during midday as can be seen in Figs. 19 and 20. During evening times, the BESS avoids an undervoltage on phase $\mathrm{S}$ by injecting power.

Figure 21 shows switch states for three boilers. Because of the single-timestep optimization a lot of switching happens during slight overvoltage times. This behavior stresses the switch relays more than necessary and reduces their lifetime. Future work should implement an MPC controller for boiler dispatch optimization. During midday, the boilers stay turned on and the internal SoC estimate rises until it approaches $100 \%$ as Fig. 22 shows. 
Fig. 19 All-in campaign: BESS active power injection

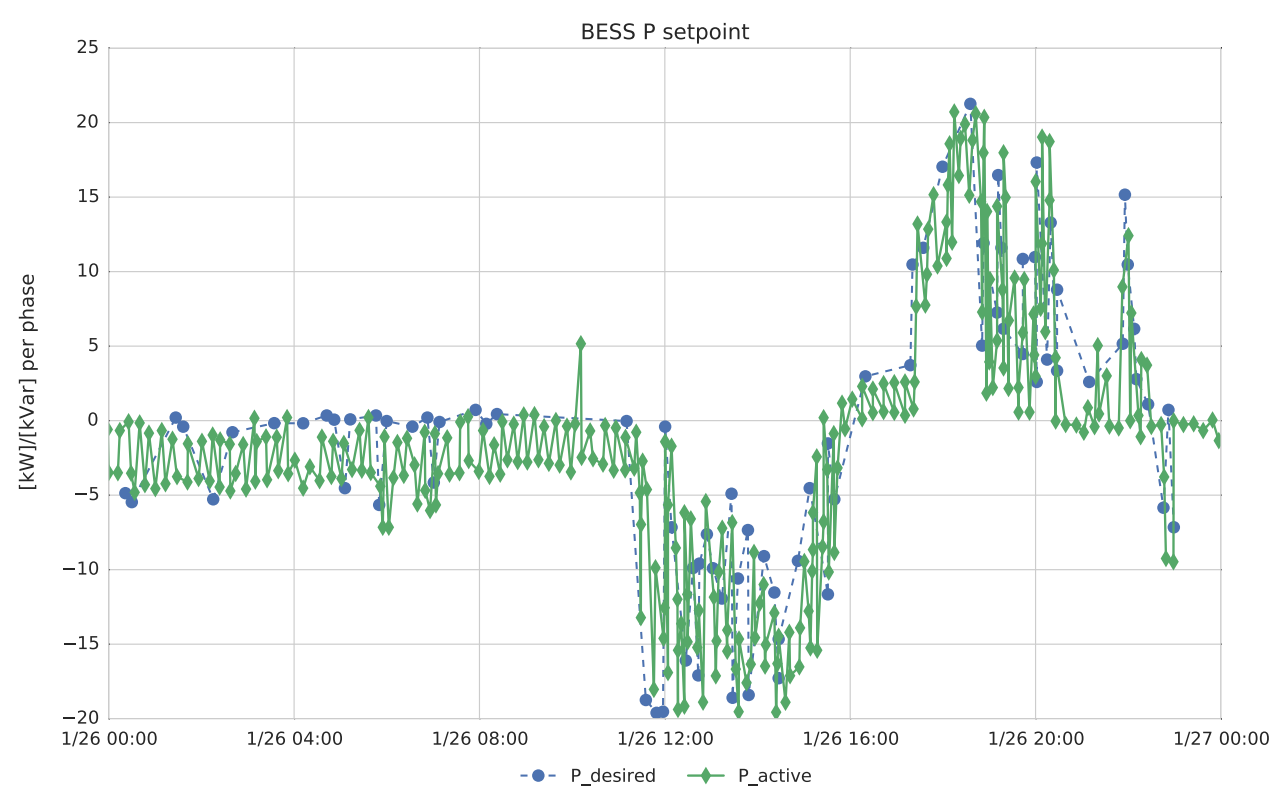

Fig. 20 All-in campaign: BESS SoC

\section{Conclusions and outlook}

The GridBox system is a novel approach for monitoring and active control of distribution grids which addresses a whole set of challenges that DSOs will face in the near future. The GridBox concept represents an open platform where the hardware and software elements are specifically developed for covering the practical needs of a DSO. The platform is designed such that it can flexibly integrate a broad set of applications in the future. In order to test the applications under typical circumstances, two different pilot regions in the distribution grids of ewz and BKW were chosen.

The rollout of 150 devices and their operation for an entire year has successfully taken place. The GridBoxes which have been installed in the medium and low voltage network measure and send realtime measurements to the GridBox Master.

Several campaigns were carried out to demonstrate the potential functionalities with a strong emphasis on evaluating state estimator performance and voltage stability optimization. It could be shown that three-phase state estimation 
Fig. 21 All-in campaign: boiler active settings

Fig. 22 All-in campaign: boiler $\mathrm{SoC}$
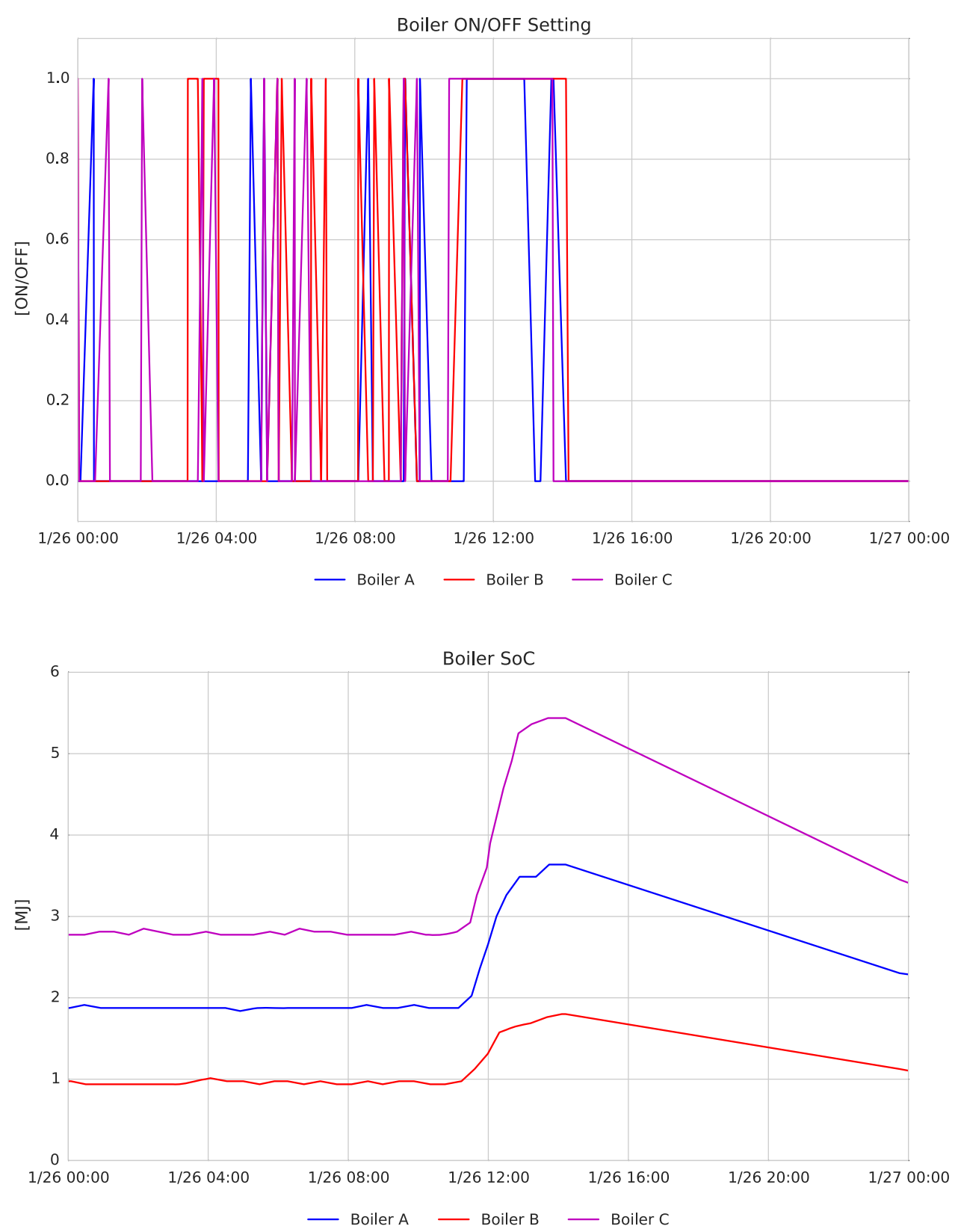

achieves an accuracy around $1 \%$ of $U_{n o m}$ and the degradation of this accuracy when using less sensors has been quantified. The optimizer campaigns showed the feasibility of jointly optimizing the dispatch of several actors of different kinds, distributed among different locations, to improve voltage stability in the low-voltage grid.

The pilot project ended by March 2016. A commercialization of the GridBox platform is planned.

Open Access This article is distributed under the terms of the Creative Commons Attribution 4.0 International License (http://creativecomm ons.org/licenses/by/4.0/), which permits unrestricted use, distribution, and reproduction in any medium, provided you give appropriate credit to the original author(s) and the source, provide a link to the Creative Commons license, and indicate if changes were made.

\section{References}

1. consentec (2012) Auswirkungen eines verstärkten Ausbaus der dezentralen Erzeugung auf die Schweizer Verteilnetze Study for the Swiss Federal Office of Energy

2. IAEW, OFFIS (2014) Moderne Verteilernetze für Deutschland, Study for the Federal Ministry for Economic Affairs and Energy in Germany 
3. BfE (2013) Swiss2G Pilot- and Demonstration Project; an innovative concept for the decentralized management of distributed energy generation, storage and consumption and consumer acceptance-Rapport annuel

4. Bolognani S, Zampieri S (2011) A gossip-like distributed optimization algorithm for reactive power flow control. In: Proc. IFAC World Congress

5. Vogel B (2016) Augen im Stromnetz. VSE-Bulletin

6. Xu O (2014) Automated topology identification in smart gridapplications. Master Thesis at SCS AG

7. Katsoulakos N (2016) Automated topology identification in smart grid. Master thesis at EPFL and SCS AG

8. Zanni L, Sarri S, Pignati M, Cherkaoui R, Paolone M (2014) Probabilistic assessment of the process-noise covariance matrix of discrete Kalman filter state estimation of active distribution networks. In: 13th probabilistic methods applied to power systems (PMAPS), Durham

9. Pignati M, Zanni L, Sarri S, Cherkaoui R, Le Boudec J-Y et al (2014) A pre-estimation filtering process of bad data for linear power systems state estimators using PMUs. In: 18th Power systems computation conference, Wrocaw

10. Hauswirth A (2015) Applications of AC optimal power flow in distribution networks. Master Thesis at ifa, ETHZ and SCS AG

11. Kwon WH, Bruckstein AM, Kailath T (1983) Stabilizing state feedback design via the moving horizon method. Int $\mathrm{J}$ Control 37:631-643

12. BfE (2016) GridBox; Netzbasierte Echtzeit-Erfassung des Verteilnetzzustandes und erste Praxistests - Schlussbericht (Publikation 291129). http://www.bfe.admin.ch/forschungnetze/01246/03569/ index.html?lang=de\&dossier_id $=06621$

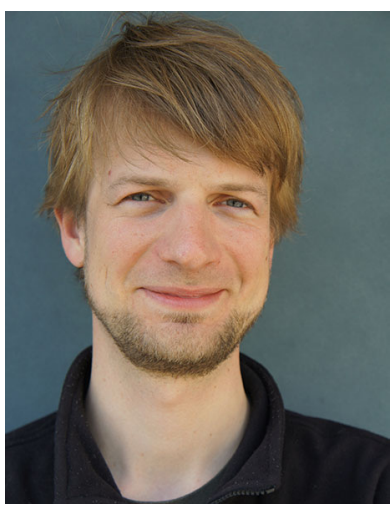

Alain Brenzikofer received his M.Sc. degree from the Federal Institute of Technology in Zurich $(\mathrm{ETH})$ in the field of Electrical Engineering and Information Technology in 2005. He Worked at Anocsys AG (2005) as research \& development engineer in the field of acoustics and signal processing and at CSEM SA (2006) in the field of optoelectronics, embedded HW/FW design and image processing algorithms. Since 2012 he works for SCS AG as project manager and research \& development engineer in the Energy and Embedded Systems department with a focus on embedded systems design and signal processing algorithms.

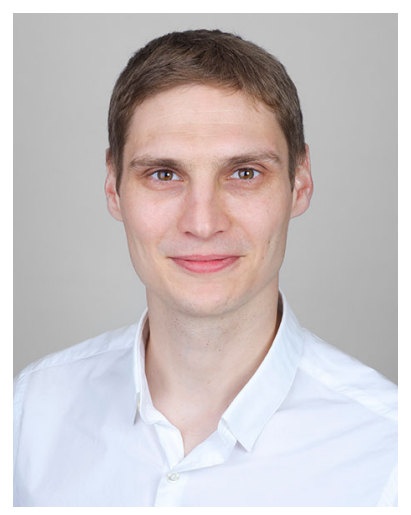

Dr. Florian Müller received his M.Sc. degree in Mechanical Engineering and his $\mathrm{PhD}$ in Computational Fluid Dynamics both from ETH Zürich. Since 2015, he is working for Supercomputing Systems AG as a software development engineer in the Energy and Embedded Systems department with a focus on energy systems simulation and optimization algorithms.

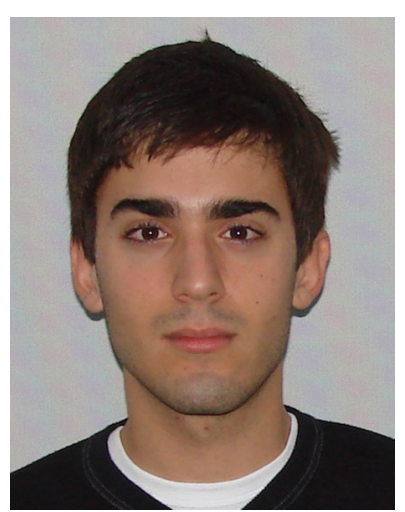

Alexandros Ketsetzis received his Diploma Degree in Electrical and Computer Engineering from the National Technical University of Athens in Greece, in 2013. Since 2014, he is a master student at the Energy Science and Technology MSc, at ETHZurich. His current interests include state estimation and optimization in electrical power grids.

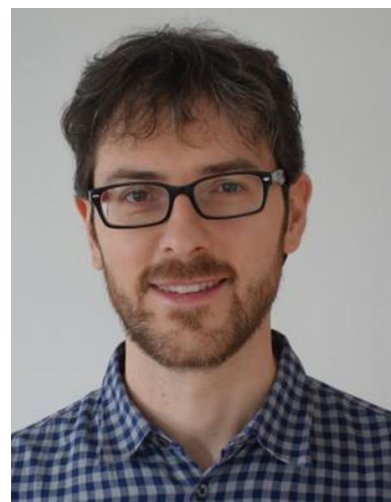

Dr. Florian Kienzle studied energy engineering at the University of Magdeburg (Germany). After that he did a PhD at the Power Systems Laboratory of ETH Zurich under the guidance of Prof. Göran Andersson. Since 2012 he has been working for ewz, the utility of the city of Zurich-initially as smart grid specialist and now as head of the team New Grid Solutions. 


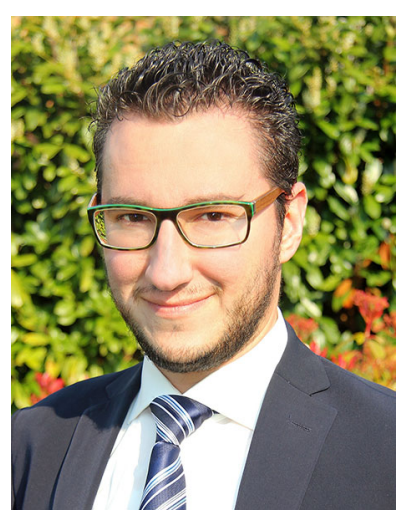

Marco Mangani Bachelor of Science, studied electrical engineering at the HSR Technical University in Rapperswil. He joined ewz in 2009 and now works in the Network Design department, where his responsibilities include studies for the integration of renewable energy sources and pilot projects for smart grids.

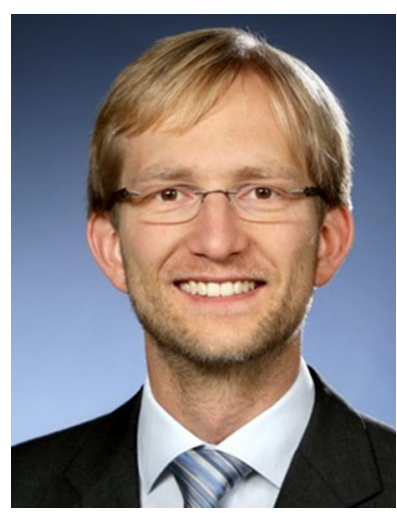

Marc Eisenreich received his degree in Business Administration and Electrical Engineering (Dipl.-Wirtsch.-Ing.) from Technische Universität Darmstadt and his degree in Engineering from Ecole Centrale de Lyon in 2008. He joined BKW's Smart Grid Engineering department in 2013 where he is responsible for pilot projects with new components and concepts in a future intelligent distribution grid.

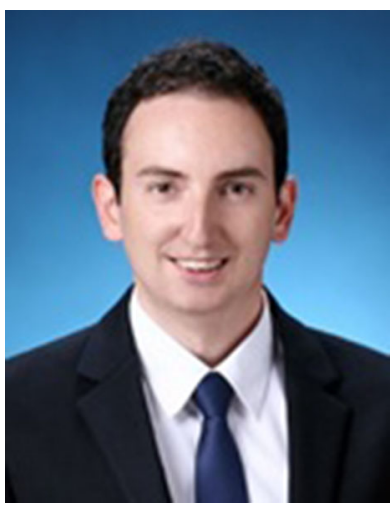

Yamshid Farhat received his M.Sc. degree from the Polytechnic University of Catalonia in Energy Engineering focusing on the Electric System. Mr. Farhat worked for his Master Thesis at the Energy Department at the Aalborg University in the field of Micro-grids. Mr. Farhat is working for BKW Energie AG since 2013 in the Smart Grid Engineering department with the focus on the development of innovative products for the Distribution Network. He is supervised by Marc Mürner, head of the Smart Grid Components group.

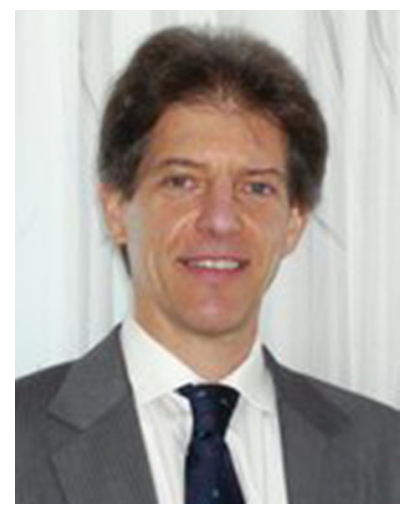

Dr. Rainer Bacher studied Electrical Engineering at the ETH Zürich (Dipl. El.-Ing. ETH, 1982; Dr. sc. techn. ETH, 1986). After employments in the US and at ETH Zürich (Switzerland) as professor, in 2002 he was at the Swiss Federal Office of Energy as section leader "Electricity Grids" and project leader of the Swiss Stromversorgungsgesetz (StromVG). He founded his own consulting company Bacher Energy LTD in 2008. There has been advising key energy policy as well as research and innovation related governmental bodies such as the Swiss Federal Office of Energy, the Swiss SmartGrids Projects funding agency, Members of the Swiss National Platform on SmartGrids, the Funding Agencies of 21+ national European governments on defining and establishing strategies for innovative Smart grids and Market based electricity/energy systems projects. He continues to be a lecturer at ETH Zürich. 